\title{
Review
}

Adrian Klak, Steven Pauwels and Pieter Vermeersch*

\section{Preanalytical considerations in therapeutic drug monitoring of immunosuppressants with dried blood spots}

https://doi.org/10.1515/dx-2018-0034

Received June 1, 2018; accepted November 22, 2018; previously published online December 18, 2018

\section{Abstract}

Background: Dried blood spots (DBSs) could allow patients to prepare their own samples at home and send them to the laboratory for therapeutic drug monitoring (TDM) of immunosuppressants. The purpose of this review is to provide an overview of the current knowledge about the impact of DBS-related preanalytical factors on TDM of tacrolimus, sirolimus and everolimus.

Content: Blood spot volume, blood spot inhomogeneity, stability of analytes in DBS and hematocrit (Hct) effects are considered important DBS-related preanalytical factors. In addition, the influence of drying time has recently been identified as a noteworthy preanalytical factor. Tacrolimus is not significantly influenced by these factors. Sirolimus and everolimus are more prone to heat degradation and exhibited variations in recovery which were dependent on Hct and drying time.

Summary and outlook: DBS-related preanalytical factors can have a significant impact on TDM for immunosuppressants. Tacrolimus is not significantly influenced by the studied preanalytical factors and is a viable candidate for DBS sampling. For sirolimus and everolimus more validation of preanalytical factors is needed. In particular, drying conditions need to be examined further, as current protocols may mask Hct-dependent effects on recovery. Further validation is also necessary for home-based selfsampling of immunosuppressants as the sampling quality is variable.

\footnotetext{
*Corresponding author: Pieter Vermeersch, Clinical Department of Laboratory Medicine, UZ Leuven, Herestraat 49, Leuven 3000 , Belgium, E-mail: pieter.vermeersch@uzleuven.be Adrian Klak and Steven Pauwels: Clinical Department of Laboratory Medicine, UZ Leuven, Leuven 3000, Belgium, E-mail: adrian.klak@gmail.com (A. Klak); steven.pauwels@jessazh.be (S.Pauwels)
}

Keywords: dried blood spot; dried blood spot testing; drying time; filter paper; hematocrit; immunosuppressants; microsampling; preanalytical phase; stability; therapeutic drug monitoring.

\section{Introduction}

The use of immunosuppressants after solid-organ transplantation contributes to the quality of life of organ recipients and offers a significant advantage in terms of survival [1]. However, the narrow therapeutic range of these drugs necessitates therapeutic drug monitoring (TDM) in order to avoid toxicity and transplant rejection [2]. TDM is often associated with a significant patient burden, as regular trips for blood sampling by a medical professional are necessary.

Dried blood spot (DBS) sampling consists of the deposition of a drop of capillary blood (preferably from the finger in adults) on filter paper by either the patient or a medical professional. The spot is then left to dry (drying times typically range from a couple of hours to overnight drying) and is shipped to the laboratory. After arrival at the laboratory, a sample is punched from the DBS, which is then followed by an extraction procedure and analysis of the sample [3]. DBS sampling has long been used for qualitative or semi-quantitative analysis such as the heelprick for the detection of inborn errors of metabolism popularized by Guthrie [4].

Recent developments, in particular the use of triple quadrupole liquid chromatography-tandem mass spectrometry (LC-MS/MS), allows quantitative analysis of a wide range of analytes due to increased sensitivity. In the past, the small amount of analyte in a punch taken from a DBS posed a problem for less sensitive assays. Consequently, interest has been rising in DBS sampling as a minimally invasive sampling method that could provide a benefit in terms of cost, ease of use and transportation, patient comfort and sample stability [3, 5, 6]. A wide variety of analytes has been studied in DBSs, including therapeutic drugs for TDM, drugs 
of abuse, serological testing, microbiology, endocrinology, clinical chemistry and genetic testing [7].

In particular, TDM of immunosuppressive agents in DBSs may be of interest because of the possibility of selfsampling. This would allow patients to prepare their own DBS at home and send them to the laboratory through regular postal services as a primary means of TDM, or to perform additional self-sampling in order to better characterize the pharmacokinetic profile of their immunosuppressive drug $[2,8-10]$.

Multiple studies have been published regarding the TDM of immunosuppressive agents in DBSs (most notably tacrolimus, sirolimus and everolimus), all of which concluded that DBS sampling is a valid alternative for venous sampling. However, most studies have been performed in controlled environments and were focused on analytical performance, often using venous sampling i.e. the application of a drop of ethylenediamine tetraacetic acid (EDTA) blood by a laboratory technician] instead of true capillary sampling. The impact of preanalytical factors specific to DBSs may be underestimated. In as many as $40 \%$ of published assays using DBSs for various analytes, not

DBS sampling process Important preanalytical factors $[6,15,16]$

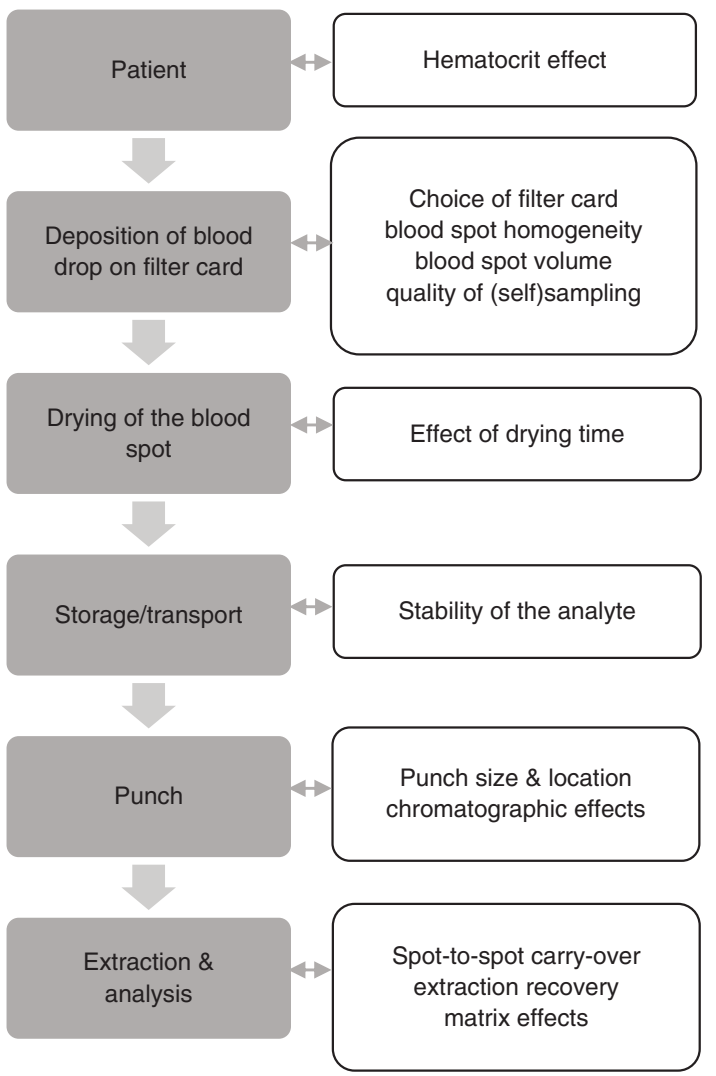

Figure 1: A summary of the important preanalytical factors for analysis of immunosuppressants with capillary DBS sampling.
A

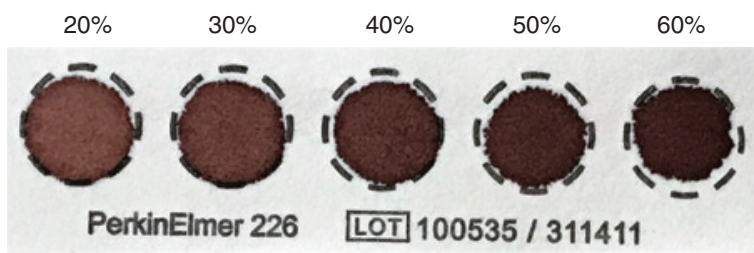

B

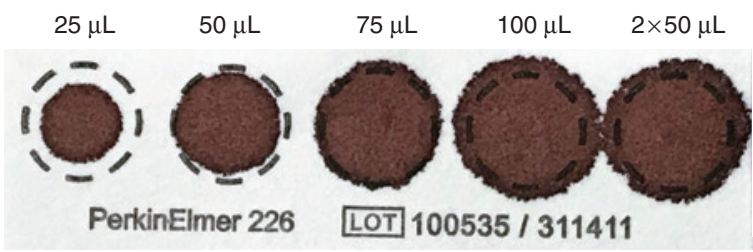

Figure 2: DBSs at different Hct levels (panel A) and using different blood volumes (panel B).

The experiment was performed using $\mathrm{K}_{3}$-EDTA anticoagulated blood from a single volunteer. For panel $A$, each circle contains $75 \mu \mathrm{L}$ $\mathrm{K}_{3}$-EDTA anticoagulated blood. For panel B, the Hct was $45 \%$.

one DBS-specific validation parameter has been studied [11]. As preanalytical errors are the main cause of errors in laboratory testing [12], more attention for DBS-specific preanalytical factors is needed, especially if self-sampling by patients is to become a reality.

Possible sources of errors in the preanalytical phase described in the literature include, among others, the quality of the self-sampled blood spot, chromatographic effects (i.e. the inhomogeneity of the spot), the influence of blood spot volume and hematocrit (Hct) and the stability of the analyte in the DBS [3,11,13,14]. Recently, more attention has also been given to the influence of variations in drying time [15]. Figure 1 gives an overview of the important preanalytical factors for TDM of immunosuppressants with DBS and Figure 2 illustrates the effect of volume and hematocrit.

We performed a thorough literature search to identify the current knowledge about the impact of DBS-related preanalytical factors on TDM of tacrolimus, sirolimus and everolimus, and to examine DBSs to what extent DBSrelated preanalytical factors are taken into account in published methods. The following preanalytical factors were studied: the influence of blood spot volume, Hct and drying time, blood spot homogeneity and influence of punch location, stability of the analyte, the influence of the DBS card type and the quality of (self-sampled) DBSs.

\section{Literature search}

The MEDLINE database has been searched with the use of PubMed for the following MeSH terms: "Dried Blood 
Spot Testing" [Mesh] combined with "Tacrolimus" [Mesh], "Sirolimus" [Mesh], "Everolimus" [Mesh]. In order to compensate for the relatively new MeSH term "Dried blood spot testing", which was only introduced in 2012, a free-text search using the phrase "Dried blood" combined with the aforementioned immunosuppressants was also performed. The last search was performed on 10/05/2018. Studies were included if they described method development and/or validation, method comparison, clinical validation or correlation study in humans for TDM of tacrolimus, sirolimus and/or everolimus in DBSs using LC-MS/MS. As ciclosporin A use is diminishing in our center and the use of this drug is projected to further decrease, we decided not to include it in our analysis.

Twenty-one methods and clinical validations were included [8, 10, 16-34]. In addition, three studies evaluating a single DBS-specific parameter in the context of immunosuppressants $[15,35]$ and three studies using TDM of tacrolimus in DBSs as a validated method for pharmacokinetic studies were included $[9,36,37]$. Two studies concerning TDM of immunosuppressants in DBSs were rejected due to the use of a method other than LC-MS/MS for analysis.

\section{Impact of DBS-related preanalytical factors}

\section{Differences between filter cards}

\section{Results of the literature search}

The most commonly used card in the included validation studies is the Whatman 903, followed by the Whatman nr. 10535097 and various Whatman FTA cards.

One method validation performed a cross-validation between Whatman 31 ET CHR paper and Whatman FTA DMPK-C cards and found no significant differences in analyte concentration [32]. In a separate study by Koster et al., five DBS cards were compared for the analysis of immunosuppressants (Whatman 31 ET CHR, Whatman FTA DMPK-C, Whatman 903, Perkin Elmer 226 and Agilent Bond Elut DMS DBS cards) [35]. All cards displayed a concentration- and Hct- dependent recovery pattern. In the low concentration ranges of all tested immunosuppressants, the effects of Hct on the formation of the blood spot did not vary substantially between the tested filter cards and the cards showed little difference in performance. In terms of recovery, Whatman 31 ET CHR cards were most affected by increasing Hct concentrations, which lead to decreasing recoveries. Whatman DMPK-C cards showed to be most resistant to the effect of increasing concentration at low Hct [35].

\section{Discussion}

A range of different filter cards is available with different physical characteristics. Typical properties of filter cards are particle retention, pore size, thickness and weight, which are properties that determine the spreading of whole blood on the paper and loading capacity [5]. While the most commonly used cards are untreated cellulose filter cards, some cards are impregnated with chemicals designed to lyse cells, inactivate pathogens or denature enzymes and other proteins [3]. Two specific cards are preferred in the literature due to experience that has been built up through the use of these cards for neonatal sampling. These cards are the Whatman 903 and Ahlstrom 226, both untreated cellulose filter cards, which are recommended by the Clinical and Laboratory Standards Institute (CLSI) guideline for neonatal screening, and are approved by the Food and Drug Administration, the Newborn Screening Quality Assurance Program (NSQAP) and the Centers for Disease Control [38, 39]. Antunes et al. reported that the Whatman 903 was used in $60 \%$ of all reviewed publications regarding TDM in DBS, which is consistent with our findings [40]. Other untreated (e.g. Whatman FTA DMPK-C) and chemically treated (e.g. Whatman FTA Elute, FTA DMPK-A and FTA DMPK-B) cards exists, however, these are not CLSI LA4-A5 compliant.

Due to their different physical characteristics, different types of filter card are likely to exhibit differences in terms of extraction recovery, matrix effects, analyte stability, chromatographic effects, Hct-related effects and volume effects [3]. Although similar results have been found in a limited cross-validation of two cards by Koster et al., the general consensus is that filter cards are not freely interchangeable and the European Bioanalysis forum (EBF) recommends that a method needs to be fully validated per filter card type and any change of card type or manufacturer requires at least a partial validation $[14,32]$.

\section{Quality of capillary (self-)sampling}

\section{Results of the literature search}

In 12 publications capillary sampling was performed in order to create the DBS, either as the primary sampling method or alongside the creation of DBSs from venous 
blood by a medical professional. The quality of the DBS was assessed by visual inspection. The most common criteria used to judge the acceptability of a DBS sample included (almost) complete and homogenous filling of the predrawn circle and a dark red color on both sides of the paper [10, 19, 41, 42].

In five publications, capillary DBS sampling was performed by a trained professional. Three of these studies reported the percentage of rejected samples due too poor blood spot quality, which consisted of $4.8 \%(n=210), 11.1 \%$ $(n=18)$, and 4.4\% $(n=91)$ rejected samples $[26,29,41]$.

Capillary sampling was performed by the patients themselves (self-sampled DBS) in seven publications. Of these studies, four took place in a hospital or clinic setting and three studies evaluated or used home-based self-sampling. In publications that reported the percentage of unsuitable samples (three studies), the percentage of unsuitable samples consisted of $1.8 \%(n=108)$ for self-sampling in a hospital setting [10] and consisted of $3.8 \%(n=26)$ and $11.6 \%(n=216)$ in a home setting $[23,34]$. Regarding the three pharmacokinetic trials using homebased self-sampled DBSs, one study reported that 3\% $(\mathrm{n}=400)$ of pharmacokinetic profiles had to be repeated due to low quality or missing samples [36].

\section{Discussion}

When DBSs are prepared by capillary sampling, the quality of the blood spot becomes an important preanalytical factor, especially in the context of home-based self-sampling. A crucial parameter for self-sampled DBSs is the amount of usable samples that patients can generate themselves. It has been found that adequate training is not a guarantee for successful sampling [5, 13]. Peck et al. showed a concerning lack of quality in blood spots for lead screening, even when sampling happened by trained professionals. Sixty-two percent of blood volumes were lower than $25 \mu \mathrm{L}$, well below the recommended $50 \mu \mathrm{L}$ per spot for Whatman 903 paper [43]. In accordance with this, Martial et al. noted that the blood samples they had obtained from children for the analysis of immunosuppressants were much smaller than they had initially anticipated [22]. In addition, Peck et al. reported that the application of multiple drops (instead of one continuous drop, as recommended by existing guidelines) was prevalent. Application of multiple drops can lead to falsely elevated values due to a more concentrated blood spot [43]. A feasibility trial for home-based selfsampling for various analyses in a motivated population of women above 50 years of age reported that only $74.1 \%$ of the received samples were taken according to instructions (although 92.9\% were suitable for at least one biochemical analysis) [44]. Recently, a feasibility trial was conducted by Al-Uzri et al. to evaluate the possibility of home-based monitoring of tacrolimus and creatinine by DBSs in children. They reported that, although $88.4 \%$ of the DBS cards were deemed suitable at first, only $80 \%$ of the blood spots on these cards were properly saturated and suitable for analysis [23].

A visual inspection of the sample is the most commonly used method to judge the acceptability of a DBS. The most important visual criteria are: (almost) complete filling of the predrawn circle, a symmetrical spread around the center and an even, dark red color on both sides of the DBS card [5]. As for the size of the blood spot, Edelbroek et al. require a blood spot that is at least larger than the punch which will be taken [5], whereas most publications require complete filling of the pre-drawn spot $[3,8]$. Furthermore, DBSs that exhibit clotting, layering, super-saturation, serum rings, visible traces of hemolysis or contamination should be rejected [3].

All studies concerning self-sampled DBSs for TDM of immunosuppressants in a hospital or clinic setting showed a sample quality comparable to that of trained personnel. In addition, the results from blood spots made by patient and trained personnel did not differ significantly [32], which was also true for blood spots made with and without nurse assistance [8]. In the case of homebased self-sampling, results were more variable. While rejection rates as low as 3\% are reported, larger feasibility trials mimicking real-life situations report $11.6 \%$ rejected DBS cards, and $20 \%$ of the accepted blood spots being ultimately unsuitable for analysis. Several other studies concerning different analytes have reported similar percentages of rejected samples (17-19\% rejected samples) [45]. This may be a realistic outcome for a patient population that has not been extensively pre-selected. A clinical trial in progress has been halted because of inadequate sample quality, suggesting that preanalytical sample quality in (self-sampled) DBSs is important [46]. Additionally, timing and adherence may be a problem, as evidenced by Al-Uzri et al., who noted that only $38 \%$ of the samples were drawn at the correct time [23].

Although self-sampled DBSs are often mentioned as a potential advantage of DBS sampling, further research is needed and a validation in a true home-based self-sampling scenario needs to be performed. Problems regarding sample quality, timing and adherence to self-sampling may occur. Self-sampled DBS at home may primarily be feasible in a selected population of motivated and capable patients. 


\section{Blood spot volume}

\section{Results of the literature search}

Seven publications included in this review have examined the effect of blood spot volume on analyte concentration in their validation protocols (Table 1). The examined volumes ranged from 15 to $100 \mu \mathrm{L}$. All seven studies noted an effect of blood volume on immunosuppressant concentration, but this effect was not deemed to be significant, with the exception of den Burger et al., who reported biases of $>15 \%$ for everolimus at low concentration and sirolimus at high concentration in $20 \mu \mathrm{L}$ spots [25]. Knapen et al. reported that the slope of the calibration curve for everolimus differed significantly between different blood volumes at a low Hct level (20\%), an effect not found at a Hct level of $40 \%$ [20]. Additionally, Hoogtanders et al. examined the effect of blood spot volume by weighing punches from blood spots made with different volumes of blood. They found that punches weighed less and thus contained less blood when $10 \mu \mathrm{L}$ of blood was used to make the blood spot versus 20 or $30 \mu \mathrm{L}$, but no difference between the two latter options was found [41].

\section{Discussion}

The volume of the blood spot has been described as an important parameter which should be included in all validation studies [40]. The EBF calls the investigation of the effects of blood spot volume an integral part of the validation of a DBS method [14]. The volume of blood that is deposited on the filter card through the use of a capillary prick is not controlled and may differ between spots on the same filter card and between patients. On cellulose-based cards (e.g. Whatman 903) an increase in blood volume will bring forth a linear increase in blood spot area. Theoretically, this should not affect the volume in a single punch, DBSs have been compared to very flat cylinders of blood which has spread continuously and homogenously through the paper. As such, a punch from a filter card should contain the same amount of blood regardless of blood spot volume [3]. However, with large differences in blood spot volume $(>20 \mu \mathrm{L})$, differences in blood volume contained in the punch and consequently concentration differences in analytes such as phenylalanine have been described [3].

In addition to the volume of the blood drop used to make the spot, the saturation properties of the paper may influence the effect of blood spot volume. For Whatman 903 paper, concentration differences in various analytes were observed with volumes up to $50 \mu \mathrm{L}$, and a stabilization between approximately $50 \mu \mathrm{L}$ and $100 \mu \mathrm{L}$ was reported, presumably due to full saturation of the paper [3]. Den Burger et al. confirmed this effect for tacrolimus, sirolimus and everolimus [25]. As a consequence, the effects of blood spot volume are dependent on paper type and should be validated together [3].

No significant interference from blood spot volume on the concentration of immunosuppressants was reported, except for sirolimus and everolimus in $20 \mu \mathrm{L}$ spots [25]. Den Burger et al. pointed out, however, that these spots would have been rejected in practice due to incomplete

Table 1: Influence of blood spot volume in method and clinical validations.

\begin{tabular}{|c|c|c|c|c|c|}
\hline Study & Card type & Range, $\mu \mathrm{L}$ & Crit. & Drug & Effect of variation in blood spot volume \\
\hline Koster et al. [32] & Whatman nr. 10535097 & $30-90$ & $15 \%$ & $\begin{array}{l}\mathrm{TaC} \\
\mathrm{SiR} \\
\mathrm{EvE}\end{array}$ & No significant effect on analyte concentration \\
\hline Sadilkova et al. [28] & Whatman 903 & $25-100$ & $\mathrm{n} / \mathrm{a}$ & $\begin{array}{l}\mathrm{TaC} \\
\mathrm{SiR}\end{array}$ & No significant effect on analyte concentration \\
\hline Den Burger et al. [25] & Whatman 903 & $20-100$ & $15 \%$ & $\begin{array}{l}\mathrm{TaC} \\
\mathrm{SiR} \\
\mathrm{EvE}\end{array}$ & $\begin{array}{l}\text { No significant effect on analyte concentration } \\
20 \mu \mathrm{L} \text { spot and HC: approx. }-17.5 \% \text { bias } \\
20 \mu \mathrm{L} \text { spot and LC: approx. }-19 \% \text { bias }\end{array}$ \\
\hline Li et al. [30] & Whatman 903 & $15-50$ & $15 \%$ & $\mathrm{TaC}$ & No significant effect on analyte concentration \\
\hline Martial et al. [22] & Whatman 903 & $20-60$ & $15 \%$ & $\mathrm{TaC}$ & No significant effect on analyte concentration \\
\hline Knapen et al. [20] & Whatman 903 & $20-50$ & $15 \%$ & EvE & At $20 \%$ Hct: difference of slope of calibration curve $>15 \%$ \\
\hline Koster et al. [21] & Whatman FTA DMPK-C & $30-70$ & $15 \%$ & $\begin{array}{l}\mathrm{TaC} \\
\mathrm{SiR} \\
\mathrm{EvE}\end{array}$ & No significant effect on analyte concentration \\
\hline
\end{tabular}

TaC, tacrolimus; SiR, sirolimus; EvE, everolimus; n/a, not available; crit., acceptability criterion; HC, high concentration; LC, low concentration. 
filling of the predrawn circle [25]. The volume of a blood drop obtained by capillary sampling has been estimated to be approximately $30 \mu \mathrm{L}[10,42]$. Knapen et al. also reported a combined effect of low Hct and blood spot volume on the slope of the calibration curve for everolimus. This effect was deemed to be primarily explained by the Hct effect [20].

The lack of clinically significant interference from blood spot volume is in accordance with a recent comprehensive review by Wagner et al., which concluded that the effect of blood spot volume appears to be negligible in a volume range of approximately $20 \mu \mathrm{L}$ around the calibration standard and accurate control of blood spot volume is not necessary [3]. A volume of $30 \mu \mathrm{L}$ is often used as a calibration standard, as it approximates the volume of a drop of blood and assures filling of the DBS spot [19].

Jager et al. recommend that a volume range of at least $15-40 \mu \mathrm{L}$ should be investigated during validation at a minimum of two concentration levels (low and high) [11]. All included studies that performed a validation for the effect of blood spot volume examined the effect at different concentrations, but only one study used a range starting from $15 \mu \mathrm{L}$. In general, more attention was given to relatively high volumes. This does not reflect clinical practice, where low volumes are more prevalent and may be more likely to cause clinically significant bias [43]. The current overrepresentation of higher volumes may mask clinically relevant effects, however, it should be noted that low blood spot volumes often lead to samples that would be rejected due to incomplete filling of the blood spot and thus would not contribute to false results.

\section{Hematocrit}

\section{Results of the literature search}

Eight publications studies examined the effect of Hct on the concentration of immunosuppressants (Table 2). The majority of method validations and all clinical validations and correlation studies reported measurable, but not necessarily clinically significant effects. In four studies, a correction was applied for the expected Hct value of the target population. Koster et al. found significant biases for sirolimus and everolimus ( $-20 \%$ and $-28 \%$, respectively) after correction at a Hct of $20 \%$ and high analyte concentration $(40 \mu \mathrm{g} / \mathrm{L})$ but found that these biases were much less pronounced when using concentrations that were more clinically relevant, and a different DBS card [21, 32]. Sirolimus showed a bias of $-15.1 \%$ at a Hct of $28 \%$ and a concentration of $3 \mathrm{ng} / \mathrm{mL}$. Knapen et al. found that bias exceeded $15 \%$ for everolimus when Hct was $20 \%$ and the concentration was 20 or $40 \mu \mathrm{g} / \mathrm{L}$ [20]. Martial et al. reported

Table 2: Influence of Hct in method and clinical validations.

\begin{tabular}{|c|c|c|c|c|c|}
\hline Study & Card type & Hct range or mean, \% & Crit & Drug & Hct effect on bias \\
\hline \multirow[t]{3}{*}{ Koster et al. [32] } & Whatman nr. 10535097 & $20-50 \%$ & $15 \%$ & $\mathrm{TaC}$ & No significant effect after correction for target $\mathrm{Hct}$ \\
\hline & & & & SiR & Hct $20 \%$ and $\mathrm{HC}:-20 \%$ bias \\
\hline & & & & EvE & Hct $20 \%$ and $\mathrm{HC}:-28 \%$ bias \\
\hline \multirow[t]{3}{*}{ Koster et al. [32] } & Whatman nr. 10535097 & Mean: & $15 \%$ & $\mathrm{TaC}$ & No significant effect (no correction) \\
\hline & & Outpatient: $38.4 \%$ & & SiR & \\
\hline & & Inpatient: $29.5 \%$ & & EvE & \\
\hline \multirow[t]{2}{*}{ Sadilkova et al. [28] } & Whatman 903 & $20-45 \%$ & $\mathrm{n} / \mathrm{a}$ & $\mathrm{TaC}$ & No significant effect (no correction) \\
\hline & & & & SiR & \\
\hline \multirow[t]{3}{*}{ Den Burger et al. [25] } & Whatman 903 & $22-41 \%$ & $15 \%$ & $\mathrm{TaC}$ & No significant effect after correction for target Hct \\
\hline & & & & SiR & \\
\hline & & & & EvE & \\
\hline Li et al. [30] & Whatman 903 & $23.2-48.6 \%$ & $15 \%$ & $\mathrm{TaC}$ & No significant effect (no correction) \\
\hline van Boekel et al. [9] & Whatman nr. 10535097 & $39 \%$ & - & $\mathrm{TaC}$ & $\begin{array}{l}\text { No significant effect on correlation with venous blood } \\
\text { (primary outcome) }\end{array}$ \\
\hline \multirow[t]{3}{*}{ Koster et al. [21] } & Whatman FTA DMPK-C & $23-53 \%$ & $15 \%$ & Tac & No significant effect \\
\hline & & & & $\mathrm{EvE}$ & \\
\hline & & & & $\operatorname{SiR}$ & Hct $28 \%$ and LC: $-15.1 \%$ bias \\
\hline Knapen et al. [20] & Whatman 903 & $20-50 \%$ & $15 \%$ & EvE & Hct $20 \%$ and $\mathrm{HC}:-18$ to $-21.9 \%$ bias \\
\hline \multirow[t]{2}{*}{ Martial et al. [22] } & Whatman 903 & $15-50 \%$ & $15 \%$ & $\mathrm{TaC}$ & Hct $15 \%$ and $M C:-24 \%$ bias \\
\hline & & & & & Hct $20 \%$ and MC: $-16 \%$ bias \\
\hline
\end{tabular}

TaC, tacrolimus; SiR, sirolimus; EvE, everolimus; n/a, not available; Hct, hematocrit; crit., acceptability criterion; HC, high concentration; MC, medium concentration; LC, low concentration. 
significant biases for tacrolimus at Hct levels of 15 and $20 \%$ at a medium concentration [22]. An evaluation of the performance of five DBS cards concluded that Hct effects were of minor impact at low concentrations of tacrolimus, sirolimus and everolimus [35].

One method validation examined the effect of Hct on the recovery of immunosuppressants. The recovery of tacrolimus was not affected by Hct. However, sirolimus and everolimus showed a decreased extraction recovery which was most pronounced at low Hct values (25\%) and high concentration $(50 \mu \mathrm{g} / \mathrm{L})$ [32]. In an evaluation of five different DBS cards, decreasing recoveries were primarily found at higher Hct values, while recovery remained constant in a Hct range of $10-40 \%$ for tacrolimus and low concentrations $(3 \mu \mathrm{g} / \mathrm{L})$ of sirolimus and everolimus. For the latter two drugs, recoveries at high concentration $(100 \mu \mathrm{g} / \mathrm{L})$ were lower than at low concentration for all Hct levels [35].

The Hct levels in blood samples of 1508 patients that underwent TDM for immunosuppressants at our center between ranged from 10.5 to $57.2 \%$. Of all patients, $95 \%$ percent had a Hct between $21.0 \%$ and $49.4 \%$.

\section{Discussion}

The influence of Hct on the results obtained through DBS sampling has been called the single most important parameter defining compound behavior and DBS assay performance and may play a significant role in the slow uptake of DBSs in clinical practice [47]. The EBF recommends that every validation of a DBS method should evaluate the effect of Hct $[14,47]$. In accordance with this, an increasing amount of studies have started including the effect of Hct in validation studies [11].

The potential effect of Hct is two-fold and may be divided into an analytical and a physiological aspect [48]. In regard to the analytical effect, Hct influences several factors such as the spread of blood on the filter card, the homogeneity of the blood spot and the drying time needed. In the classic Hct-associated interference, a higher Hct will lead to a smaller, denser blood spot on cellulose-based paper [48]. The blood spot area decreased by $15 \%$ on Whatman 903 paper when Hct was increased from 30 to $70 \%$. A punched-out sample from a blood spot from a patient with a high Hct will therefore contain more blood, leading to an overestimation of the true concentration. In Whatman 903 paper, a blood volume difference of $35 \%$ was reported in punches taken from blood spots created with blood with a Hct of $20-80 \%$ [49].

The analytical effects of Hct are, however, not limited to the increased blood volume per spot. Changes in Hct influence chromatographic effects and they may interfere in analyte extraction from the blood spot. A high Hct may lead to the formation of a barrier after drying, which interferes in extraction of the analyte [48]. In addition, a low Hct has been found to decrease recovery of certain analytes such as immunosuppressants as well [32].

The physiological effect of Hct consists of its effect on the blood-to-plasma ratio of certain analytes. As TDM is often based on measurements in serum or plasma, results obtained from whole blood need to be converted. This conversion relies on the blood-to-plasma ratio, which is dependent on Hct, especially for analytes that primarily reside in the plasma fraction and do not bind or enter erythrocytes [48]. However, the immunosuppressive drugs included in this review, i.e. tacrolimus, sirolimus and everolimus, are already measured and reported in whole blood and trough levels are measured before the administration of the next dose, minimizing the effects of the speed of drug distribution between capillary and venous blood. Consequently, no significant difference between venous samples and DBS samples is expected in this regard [5].

In general, the effect of Hct on the concentration of immunosuppressants was not deemed to be significant in clinical practice or could be corrected relatively easily. Tacrolimus was least affected by Hct. Only one of the included studies reported a significant bias at the very low end of clinically relevant Hct levels. The lack of Hct effect in regard to tacrolimus has been noted to be sufficiently proven by Veenhof et al., such that no additional correction for Hct was deemed to be necessary in their method validation [29].

Sirolimus and everolimus are more susceptible to interference from Hct. A bias that persisted after the correction has been noted at low Hct levels (20\%) and high concentrations $(40 \mu \mathrm{g} / \mathrm{L})$ [25]. Additionally, a Hct-dependent recovery has been found, which may be related to the formation of hydrogen bridges between the analytes and the cellulose paper. A low Hct combined with a high concentration may increase the non-erythrocyte bound drug fraction, which is then free to form hydrogen bonds with the cellulose paper. As Koster et al. noted, this effect was found at Hct levels and drug concentrations which are very rare in clinical practice [15]. For tacrolimus, sirolimus and everolimus, it has been suggested that DBS analysis of trough levels is possible without any Hct correction in a range of $20-60 \%$ Hct, which corresponds well to the distribution of Hct in patients undergoing TDM for immunosuppressants at our center [35].

The effect of Hct was most pronounced in the setting of method validation, where extremes for both Hct and 
analyte concentration can be produced. The lack of a significant Hct effect in a clinical setting can partly be explained by the infrequent occurrence of these extremes in clinical practice.

Several methods have been proposed to either avoid or correct for any Hct-related effects that may be present. The simplest method to avoid the effect is to use whole blood spot analysis, coupled with volumetric application of the blood spot [3]. This, however, adds complexity to the sampling process and lessens the applicability of self-sampling in a home-based setting. Recently, single-use devices have been developed which simplify the volumetric sampling process and could enable volumetric self-sampling. De Kesel et al. reported that volumetric absorptive microsampling (VAM) allows for a straightforward volumetric sampling of a drop of blood and assists in eliminating the effect of Hct [50].

For the correction of any Hct effects that may occur, several options exist. A correction for the estimated Hct of the target population is a simple but an effective approach to minimize the Hct effect for a large part of the study population [48]. This approach was used to good effect in several studies included in this review. In these studies, additional measurements were performed in order to measure the Hct of the target population. In clinical practice, available historical data could be used, but this approach needs to be validated. Estimation of the Hct of the patient is also possible by measurement of potassium in the DBS [51] or a photometric measurement of hemoglobin content [52]. A correction can then be applied, which enables an accurate measurement, even for Hct-sensitive analytes [53]. A novel extraction method which uses heated flow-through desorption has been studied for immunosuppressants in DBS and has been found to eliminate Hct effects [33]. However, only one publication currently exists using this method for immunosuppressants [33].

It is important to note that the impact of Hct cannot be evaluated alone. Factors such as blood spot volume and punch location interact with each other and with the Hct effect. Furthermore, the impact of the Hct depends on the analyte and the paper material [3]. The combined effect of Hct, blood spot volume and punch location should be evaluated.

\section{Drying time}

\section{Results of the literature search}

Drying time in the studies ranged from "at least 10 min" [34] or (at least) $3 \mathrm{~h}[28,31]$ to overnight drying [25, 30, 32,
$41,54]$ and a drying time of one to several days [29]. None of the method or clinical validation studies included the effect of drying time in their protocol.

In a separate study, Koster et al. examined the influence of drying time on the recovery of immunosuppressive drugs on Whatman FTA DMPK-C filter cards [15]. Variation in drying time (3-48 h) had no effect on tacrolimus recovery, but significantly impacted the recovery of sirolimus and everolimus. The recovery of these immunosuppressants decreased significantly between 3 and $24 \mathrm{~h}$ of drying time, after which it stabilized. This effect was Hct-dependent and was more pronounced at low Hct levels (10\%) combined with a high concentration $(100 \mu \mathrm{g} / \mathrm{L})$ where the decline in recovery between 3 and $24 \mathrm{~h}$ was $24 \%$ for sirolimus and $26 \%$ for everolimus [15].

\section{Discussion}

Standard protocols recommended by the CLSI for newborn screening with DBSs, the area in which most clinical expertise with DBSs has been gathered, recommend a drying time of at least $3 \mathrm{~h}$ at room temperature, shielded from direct sunlight [38]. Residual humidity may cause bacterial or fungal growth and can alter the elution time of the specimen [55]. Several factors, such as Hct and blood spot volume influence the drying time a blood spot may need [14]. The EBF recommends that every full and partial DBS validation include an evaluation of drying conditions (i.e. drying time and temperature) [14].

The drying time varied significantly between the studies included for review and none of the included method and clinical validations included an evaluation of the effect of drying time. In a separate study, Koster et al. examined this effect in detail and concluded that sirolimus and everolimus are susceptible to Hct-dependent changes in recovery when drying times exceed $3 \mathrm{~h}$ [15]. This effect may be explained by the formation of hydrogen bonds between sirolimus and everolimus and the cellulose paper [56]. Koster et al. noted that when drying time is only $3 \mathrm{~h}$, this may mask the effects of Hct and may provide misleading results for sirolimus and everolimus, which underestimate the effects of Hct. A drying time of $24 \mathrm{~h}$ for sirolimus and everolimus was recommended to achieve a stable, albeit lower recovery [15]. Although the concentrations used in these studies were far above the expected concentrations in a reallife setting, the effect of drying time for sirolimus and everolimus needs further attention in method- and clinical validation to establish whether clinically relevant interference can occur. 


\section{Stability}

\section{Results of the literature search}

Fourteen publications have examined the stability of immunosuppressants in DBSs. No studies reported significant degradation of tacrolimus at room temperature within the measured timeframes, which ranged from 5 days to 8 months [18, 22, 32]. When refrigerated or frozen, long-term stability for at least 1 week to 168 days has been demonstrated [16, 25]. At higher temperatures, tacrolimus was found to be stable at $37^{\circ} \mathrm{C}$ for at least $4-28$ days $[18,30$, $32,41]$, at least 5 days at $60^{\circ} \mathrm{C}[28]$ and at least 1 day at $50^{\circ} \mathrm{C}$ and $70^{\circ} \mathrm{C}[30,41]$. Sirolimus and everolimus were reported to share the same stability at low and room temperature as tacrolimus but were found to be more susceptible to long-term degradation in warm temperature conditions, as they showed degradation at $37^{\circ} \mathrm{C}$ over a 4-week period [32]. Additionally, sirolimus showed degradation within $24 \mathrm{~h}$ at $60^{\circ} \mathrm{C}$ [28] and everolimus was deemed to be unstable in DBSs at $70^{\circ} \mathrm{C}[19]$.

Two studies examined the stability of DBS samples for the analysis of tacrolimus in long-distance shipping conditions. Hoogtanders et al. shipped patient DBS samples from Japan to the Netherlands and found no degradation of the samples [8]. Cheung et al. sent samples from Hong Kong to the Netherlands and found the samples to be stable during long-distance transport [10].

\section{Discussion}

DBSs have been described as a stable medium for a variety of analytes, even without the need for refrigeration or special precautions $[3,5,13]$. DBSs improve the stability of most analytes in comparison to plasma, serum or whole blood samples, possibly due to removal of esterase activity upon drying [40]. Omeprazole and nifedipine are examples of drugs that are stabilized in the DBSs and consequently are far more stable than in plasma [3]. Biomarkers such as acylcarnitines and several amino acids are stable for several weeks at room temperature [3] and nucleic acids may be stable for several months or even more than 1 year [7]. However, stability needs to be investigated on a case-by-case basis, as notable exceptions exist. Several analytes, such as ornithine, sarcosine and serine show a significant decrease after 1 day at room temperature [3].

It is important to measure analyte stability in several temperature conditions, as the possibility of self-sampling at home includes transport and handling. Temperature in post boxes can be $20^{\circ} \mathrm{C}$ above outside temperature and may reach $60^{\circ} \mathrm{C}$ [57].

DBSs have proven to be a stable medium for immunosuppressants for both long- and short-term storage in a variety of temperature conditions. Tacrolimus showed an excellent stability, even at high temperatures. Sirolimus and everolimus are stable at room temperature but were reported to be more susceptible to higher temperatures, which may present problems during transport in warm months. Although the stability of immunosuppressants in DBS has been well documented, more validation on interfering factors such as drying time is needed.

\section{Spot homogeneity and punch location}

\section{Results of the literature search}

Two studies examined the effect of punch location. Den Burger et al. found no significant concentration difference at different punch locations (central versus peripheral) for tacrolimus, sirolimus and everolimus [25]. Li et al. found no significant chromatographic effects for tacrolimus on DBSs when comparing central and peripheral punches [30].

Sadilkova et al. unevenly soaked filter paper with $4 \mathrm{~mL}$ of patient blood and punched 218 -mm punches spread throughout the card in order to study the effect of punch location. The coefficient of variation for tacrolimus and sirolimus concentrations between the spots was $<15 \%$ [28].

\section{Discussion}

Due to chromatographic effects, i.e. the inhomogeneous spread of blood through the filter paper, the location of a punch may have an effect on the concentration of the analyte [3]. Chromatographic effects can be influenced by the Hct, filter paper, humidity, drying conditions and filter card position while drying [40]. A "volcano pattern" has been described for a number of analytes (a higher concentration between the center and the edge [58]) which is more pronounced on pre-treated cards (e.g. FTA Elute) than on untreated cellulose cards [3]. In addition, a "coffee-stain" effect is seen as well, which consists of the accretion of solid particles, e.g. red blood cells, at the edge of the blood spot [58]. An effect of the coffee-stain effect on analytes which are primarily located in erythrocytes has been described. The concentration of lead was increased 1.5 times when the punch was located within $1 \mathrm{~mm}$ of the blood spot edge when compared to the center [43]. 
None of the studies that investigated the effect of punch location found a significant effect on the concentration of tacrolimus, sirolimus and everolimus. The lack of significant effects of punch location is in accordance with guidelines by the EBF, which mention that spot homogeneity usually does not seem to be an issue and with Jager et al., who reviewed the validation of DBS methods and found no reports of significant interference because of spot inhomogeneity [11, 14].

The impact of any chromatographic effects that may be present is influenced by the size of the punch and the DBS. A large punch will punch out overlapping areas and will compensate for possible differences [3]. The lack of a chromatographic effect in the reviewed publications may partly be explained by the large punch sizes. For example, den Burger et al. used an 8-mm punch which punched out approximately $67 \%$ of the entire spot [25]. The EBF recommends taking a punch that is big enough to be a representative sample and/or to always punch from the same location on the blood spots. The punch size in the reviewed publications ranged from 6 to $10 \mathrm{~mm}$ and, when specified, all punches were made from the center of the blood spot. For analytes which are primarily located in erythrocytes, such as immunosuppressants, it may be important to avoid the edge of the blood spot, as red blood cell accretion at the perimeter has been noted. In this regard, it is crucial to reject samples with insufficient volume. Keeping the EBF recommendations in mind, no significant impact from spot inhomogeneity is to be expected.

\section{Summary}

DBS sampling is a promising sampling method with benefits in terms of ease of use, patient comfort, stability and cost. The possibility of self-sampling by the patient is interesting, especially in the context of immunosuppressant TDM. However, DBS-related preanalytical factors are not always part of the method validation in published methods: out of 21 method validations, seven publications (33\%) studied the influence of blood spot volume, 14 $(66 \%)$ the stability of immunosuppressants in DBSs, nine (42\%) the influence of Hct, 3 (14\%) the effect of punch location and no method validations included the influence of drying time.

Tacrolimus was not significantly influenced by blood spot volume, Hct effects, blood spot inhomogeneity and drying times. Furthermore, tacrolimus was found to be sufficiently stable at low and high temperatures, as no significant degradation was noted after 5 days at $60^{\circ} \mathrm{C}$. This lack of interference makes tacrolimus a viable candidate for DBS sampling, even in a home-based setting. Several pharmacokinetic trials have already used homebased self-sampled DBS of tacrolimus as an established method, demonstrating the feasibility of this method.

Sirolimus and everolimus were more prone to interference from the studied preanalytical factors. They exhibited significant bias in blood spots with low volume, although the clinical impact is minimal, as these spots would have been rejected due to insufficient filling. High temperatures significantly impacted the stability of these compounds at $60^{\circ} \mathrm{C}$ and $70^{\circ} \mathrm{C}$, temperatures which can be reached in postal boxes during summer months.

Furthermore, sirolimus and everolimus were more susceptible to Hct-related effects than tacrolimus, as they exhibited variations in recovery (up to $-26 \%$ ) which were dependent on Hct, concentration and drying time. A significant Hct-dependent bias on concentration was also found at low Hct and high concentration. Although the impact of Hct seems to be minimal in clinical practice, where extremes of Hct and concentration are rare, further validation of these preanalytical factors is needed before their use in DBSs can become widespread. In particular, drying conditions for these compounds need to be examined further, as current protocols for drying time may mask Hct-dependent effects on recovery.

Finally, although the possibility of home-based selfsampling of DBSs is often reported, it has not been extensively studied. Several publications show promising results, but the quality of blood spot made by patients at home is variable (the amount of rejected samples ranges from $3 \%$ up to $11.6 \%$ ) and problems with timing and adherence to self-sampling have been noted. Self-sampling at home may primarily be reserved for a selected population of patients and further validation in a home setting and development of protocols for patient training and selection are needed.

Author contributions: The author has accepted responsibility for the entire content of this submitted manuscript and approved submission.

Research funding: None declared.

Employment or leadership: Pieter Vermeersch is a senior clinical investigator of the Research Foundation, Flanders (Belgium) (FWO).

Honorarium: None declared.

Competing interests: The funding organization(s) played no role in the study design; in the collection, analysis, and interpretation of data; in the writing of the report; or in the decision to submit the report for publication. 


\section{References}

1. van Sandwijk MS, Bemelman FJ, Ten Berge IJ. Immunosuppressive drugs after solid organ transplantation. Neth J Med 2013;71:281-9.

2. McShane AJ, Bunch DR, Wang S. Therapeutic drug monitoring of immunosuppressants by liquid chromatography-mass spectrometry. Clin Chim Acta 2016;454:1-5.

3. Wagner M, Tonoli D, Varesio E, Hopfgartner G. The use of mass spectrometry to analyze dried blood spots. Mass Spectrom Rev 2016;35:361-438.

4. Guthrie R, Susi A. A simple phenylalanine method for detecting phenylketonuria in large populations of infants. Pediatrics 1963;32:338-43.

5. Edelbroek PM, van der Heijden J, Stolk L. Dried blood spot methods in therapeutic drug monitoring: methods, assays, and pitfalls. Ther Drug Monit 2009;31:327-36.

6. Martial LC, Aarnoutse RE, Schreuder MF, Henriet SS, Brüggemann RJ, Joore MA. Cost evaluation of dried blood spot home sampling as compared to conventional sampling for therapeutic drug monitoring in children. PLoS One 2016;11:e0167433.

7. Lehmann S, Delaby C, Vialaret J, Ducos J, Hirtz C. Current and future use of "dried blood spot" analyses in clinical chemistry. Clin Chem Lab Med 2013;51:1897-909.

8. Hoogtanders K, van der Heijden J, Christiaans M, van de Plas A, van Hooff J, Stolk L. Dried blood spot measurement of tacrolimus is promising for patient monitoring. Transplantation 2007;83:237-8.

9. Van Boekel GA, Aarnoutse RE, Hoogtanders KE, Havenith TR, Hilbrands LB. Delayed trough level measurement with the use of prolonged-release tacrolimus. Transpl Int 2015;28:314-8.

10. Cheung CY, Van Der Heijden J, Hoogtanders K, Christiaans M, Liu YL, Chan YH, et al. Dried blood spot measurement: application in tacrolimus monitoring using limited sampling strategy and abbreviated AUC estimation. Transpl Int 2008;21:140-5.

11. Jager NG, Rosing H, Schellens JH, Beijnen JH. Procedures and practices for the validation of bioanalytical methods using dried blood spots: a review. Bioanalysis 2014;6:2481-514.

12. Plebani M. Quality indicators to detect pre-analytical errors in laboratory testing. Clin Biochem Rev 2012;33:85-8.

13. Wilhelm AJ, den Burger JC, Swart EL. Therapeutic drug monitoring by dried blood spot: progress to date and future directions. Clin Pharmacokinet 2014;53:961-73.

14. Timmerman P, White S, Globig S, Lüdtke S, Brunet L, Smeraglia J. EBF recommendation on the validation of bioanalytical methods for dried blood spots. Bioanalysis 2011;3:1567-75.

15. Koster RA, Botma R, Greijdanus B, Uges DR, Kosterink JG, Alffenaar J-W, et al. The influence of the dried blood spot drying time on the recoveries of six immunosuppressants. J Appl Bioanal 2015;1:116-22.

16. Shokati T, Bodenberger N, Gadpaille H, Schniedewind B, Vinks AA, Jiang W, et al. Quantification of the immunosuppressant tacrolimus on dried blood spots using LC-MS/MS. J Vis Exp 2015; e52424.

17. Hinchliffe E, Adaway J, Fildes J, Rowan A, Keevil BG. Therapeutic drug monitoring of ciclosporin $A$ and tacrolimus in heart lung transplant patients using dried blood spots. Ann Clin Biochem An Int J Biochem Lab Med 2014;51:106-9.
18. Dickerson JA, Sinkey M, Jacot K, Stack J, Sadilkova K, Law YM, et al. Tacrolimus and sirolimus in capillary dried blood spots allows for remote monitoring. Pediatr Transplant 2015;19:101-6.

19. van der Heijden J, de Beer Y, Hoogtanders K, Christiaans M, de Jong GJ, Neef $C$, et al. Therapeutic drug monitoring of everolimus using the dried blood spot method in combination with liquid chromatography-mass spectrometry. J Pharm Biomed Anal 2009;50:664-70.

20. Knapen LM, de Beer Y, Brüggemann RJ, Stolk LM, de Vries F, Tjan-Heijnen VC, et al. Development and validation of an analytical method using UPLC-MS/MS to quantify everolimus in dried blood spots in the oncology setting. J Pharm Biomed Anal 2018;149:106-13.

21. Koster RA, Veenhof H, Botma R, Hoekstra AT, Berger SP, Bakker SJ, et al. Dried blood spot validation of five immunosuppressants, without hematocrit correction, on two LC-MS/MS systems. Bioanalysis 2017;9:553-63.

22. Martial LC, Hoogtanders KE, Schreuder MF, Cornelissen EA, Van Der Heijden J, Joore MA, et al. Dried blood spot sampling for tacrolimus and mycophenolic acid in children: analytical and clinical validation. Ther Drug Monit 2017;39:412-21.

23. Al-Uzri A, Freeman KA, Wade J, Clark K, Bleyle LA, Munar M, et al. Longitudinal study on the use of dried blood spots for home monitoring in children after kidney transplantation. Pediatr Transplant 2017;21:1-11.

24. Willemsen AE, Knapen LM, de Beer YM, Brüggemann RJ, Croes $\mathrm{S}$, van Herpen $\mathrm{CM}$, et al. Clinical validation study of dried blood spot for determining everolimus concentration in patients with cancer. Eur J Clin Pharmacol 2018;74:465-71.

25. Den Burger JC, Wilhelm AJ, Chahbouni A, Vos RM, Sinjewel A, Swart EL. Analysis of cyclosporin A, tacrolimus, sirolimus, and everolimus in dried blood spot samples using liquid chromatography tandem mass spectrometry. Anal Bioanal Chem 2012;404:1803-11.

26. Koop DR, Bleyle LA, Munar M, Cherala G, Al-Uzri A. Analysis of tacrolimus and creatinine from a single dried blood spot using liquid chromatography tandem mass spectrometry. J Chromatogr B Analyt Technol Biomed Life Sci 2013;926:54-61.

27. Hinchliffe E, Adaway JE, Keevil BG. Simultaneous measurement of cyclosporin $A$ and tacrolimus from dried blood spots by ultra high performance liquid chromatography tandem mass spectrometry. J Chromatogr B Anal Technol Biomed Life Sci 2012;883-884:102-7.

28. Sadilkova K, Busby B, Dickerson JA, Rutledge JC, Jack RM. Clinical validation and implementation of a multiplexed immunosuppressant assay in dried blood spots by LC-MS/MS. Clin Chim Acta 2013;421:152-6.

29. Veenhof H, Koster RA, Alffenaar J-W, Berger SP, Bakker SJ, Touw DJ. Clinical validation of simultaneous analysis of tacrolimus, cyclosporine a and creatinine in dried blood spots in kidney transplant patients. Transplantation 2017;101:1727-33.

30. Li Q, Cao D, Huang Y, Xu H, Yu C, Li Z. Development and validation of a sensitive LC-MS/MS method for determination of tacrolimus on dried blood spots. Biomed Chromatogr 2013;27:327-34.

31. Rao RN, Maurya PK, Ramesh M, Srinivas R, Agwane SB. Development of a validated high-throughput LC-ESI-MS method for determination of sirolimus on dried blood spots. Biomed Chromatogr 2010;24:1356-64. 
32. Koster RA, Alffenaar J-W, Greijdanus B, Uges DR. Fast LC-MS/MS analysis of tacrolimus, sirolimus, everolimus and cyclosporin $A$ in dried blood spots and the influence of the hematocrit and immunosuppressant concentration on recovery. Talanta 2013;115:47-54.

33. Hempen CM, Maarten Koster EH, Ooms JA. Hematocrit-independent recovery of immunosuppressants from DBS using heated flow-through desorption. Bioanalysis 2015;7:2019-29.

34. van Boekel GA, Donders AR, Hoogtanders KE, Havenith TR, Hilbrands LB, Aarnoutse RE. Limited sampling strategy for prolonged-release tacrolimus in renal transplant patients by use of the dried blood spot technique. Eur J Clin Pharmacol 2015;71:811-6.

35. Koster RA, Botma R, Greijdanus B, Uges DR, Kosterink JG, Touw DJ, et al. The performance of five different dried blood spot cards for the analysis of six immunosuppressants. Bioanalysis 2015;7:1225-35.

36. Stifft F, Stolk LM, Undre N, van Hooff JP, Christiaans MH. Lower variability in 24-hour exposure during once-daily compared to twice-daily tacrolimus formulation in kidney transplantation. Transplantation 2013;97:1.

37. van Boekel GA, Aarnoutse RE, van der Heijden JJ, Hoogtanders KE, Hilbrands LB. Effect of mild diarrhea on tacrolimus exposure. Transplantation 2012;94:763-7.

38. Hannon WH, De Jesús VR. Blood collection on filter paper for newborn screening programs. Approved Standard - Sixth Edition, 2013. CLSI document NBS01-A6.

39. Zakaria R, Allen KJ, Koplin JJ, Roche P, Greaves RF. Advantages and challenges of dried blood spot analysis by mass spectrometry across the total testing process. EJIFCC 2016;27:288-317.

40. Antunes MV, Charão MF, Linden R. Dried blood spots analysis with mass spectrometry: potentials and pitfalls in therapeutic drug monitoring. Clin Biochem 2016;49:1035-46.

41. Hoogtanders K, van der Heijden J, Christiaans M, Edelbroek P, van Hooff JP, Stolk LM. Therapeutic drug monitoring of tacrolimus with the dried blood spot method. J Pharm Biomed Anal 2007;44(3 Spec. Iss.):658-64.

42. Wilhelm AJ, Klijn A, den Burger JC, Visser OJ, Veldkamp AI, Janssen JJ, et al. Clinical validation of dried blood spot sampling in therapeutic drug monitoring of ciclosporin A in allogeneic stem cell transplant recipients: direct comparison between capillary and venous sampling. Ther Drug Monit 2013;35:92-5.

43. Peck HR, Timko DM, Landmark JD, Stickle DF. A survey of apparent blood volumes and sample geometries among filter paper bloodspot samples submitted for lead screening. Clin Chim Acta 2009;400:103-6.

44. Sakhi AK, Bastani NE, Ellingjord-Dale M, Gundersen TE, Blomhoff R, Ursin G. Feasibility of self-sampled dried blood spot and saliva samples sent by mail in a population-based study. BMC Cancer 2015;15:265.

45. Tanna S, Lawson G. Self-sampling and quantitative analysis of DBS: can it shift the balance in over-burdened healthcare systems? Bioanalysis 2015;7:1963-6.

46. To Validate an Analytical Method to Measure Concentration of Tacrolimus in Blood Taken From Finger Pricks - Full Text View - ClinicalTrials.gov [Internet]. [cited 2017 Feb 8]. https://clinicaltrials.gov/ct2/show/NCT02377609.

47. Timmerman P, White S, Cobb Z, de Vries R, Thomas E, van Baar $B$. Update of the EBF recommendation for the use of DBS in regulated bioanalysis integrating the conclusions from the EBF DBS-microsampling consortium. Bioanalysis 2013;5:2129-36.

48. De Kesel PM, Sadones N, Capiau S, Lambert WE, Stove CP. Hemato-critical issues in quantitative analysis of dried blood spots: challenges and solutions. Bioanalysis 2013;5:2023-41.

49. Denniff $P$, Spooner $N$. The effect of hematocrit on assay bias when using DBS samples for the quantitative bioanalysis of drugs. Bioanalysis 2010;2:1385-95.

50. De Kesel PM, Lambert WE, Stove CP. Does volumetric absorptive microsampling eliminate the hematocrit bias for caffeine and paraxanthine in dried blood samples? A comparative study. Anal Chim Acta 2015;881:65-73.

51. Capiau S, Stove VV, Lambert WE, Stove CP. Prediction of the hematocrit of dried blood spots via potassium measurement on a routine clinical chemistry analyzer. Anal Chem 2013;85:404-10.

52. Capiau S, Wilk LS, Aalders MC, Stove CP. A novel, nondestructive, dried blood spot-based hematocrit prediction method using noncontact diffuse reflectance spectroscopy. Anal Chem 2016;88:6538-46

53. De Kesel PM, Capiau S, Stove VV, Lambert WE, Stove CP. Potassium-based algorithm allows correction for the hematocrit bias in quantitative analysis of caffeine and its major metabolite in dried blood spots. Anal Bioanal Chem 2014;406:6749-55.

54. Wilhelm AJ, den Burger JC, Vos RM, Chahbouni A, Sinjewel A. Analysis of cyclosporin $A$ in dried blood spots using liquid chromatography tandem mass spectrometry. J Chromatogr B Anal Technol Biomed Life Sci 2009;877:1595-8.

55. Mei JV, Alexander JR, Adam BW, Hannon WH. Use of filter paper for the collection and analysis of human whole blood specimens. J Nutr 2001;131:1631S-6S.

56. Koster RA, Alffenaar J-W, Botma R, Greijdanus B, Uges DR, Kosterink JG, et al. The relation of the number of hydrogen-bond acceptors with recoveries of immunosuppressants in DBS analysis. Bioanalysis 2015;7:1717-22.

57. Black J, Layloff T. Summer of 1995-Mailbox temperature excursions in St Louis. Pharmacopeial Forum 1996;22:3305.

58. Lenk G, Hansson J, Beck O, Roxhed N. The effect of drying on the homogeneity of DBS. Bioanalysis 2015;7:1977-85. 